\title{
ANTECEDENTES SOBRE LA CAPACIDAD DE RETOÑACION DE ALGUNAS ESPECIES DEL GENERO EUCALYPTUS EN CHILE
}

\author{
J. Antonio Prado D. (*) \\ J. Carlos Bañados $(*)$ \\ Andrés Bello D. $\left({ }^{*}\right)$
}

\section{RESUMEN}

Se analiza la capacidad de retoñación de 5 especies del género Eucalyptus, $\boldsymbol{E}$. delegatensis, E. globulus ssp. globulus, E. nitens, E. regnans y E. viminalis.

Un año después de la corta sólo $\boldsymbol{E}$. regnans no presentaba una retoñación suficiente como para asegurar la siguiente rotación de monte bajo. Las otras especies retoñaron bien, incluso $\boldsymbol{E}$. nitens $y \boldsymbol{E}$. delegatensis, especies que tradicionalmente se han considerado con ciertas limitaciones para rebrotar despues de la corta.

Retoñación, Eucalyptus.

\section{ABSTRACT}

Coppicing ability of 5 Eucalyptus species including E. delegatensis, E. globulus $s s p$. globulus, $E$. nitens, $E$. regnans y $E$. viminalis, is analized.

One year after the trees were cut, only $\boldsymbol{E}$. regnans did not coppice abundantly enough to assure the next coppice rotation. The other species coppiced well, including $\dot{E}$. nitens and $E$. delegatensis, species which are considered having a poor coppice ability.

Coppice, Eucalyptus. 


\section{INTRODUCCION}

La creciente demanda mundial por madera de fibra corta ha despertado un gran interés por la plantación con especies del género Eucalyptus.

Por su gran adaptabilidad a diversas condiciones de suelo y clima, en Chile existen varias especies que presentan altas tasas de crecimiento y que en muchos casos constituyen alternativas para la plantación en un determinado lugar.

La selección de la especie adecuada para un sitio en particular dependerá de varios factores, entre los que se destacan los aspectos climáticos y el objetivo de la plantación.

Cuando el objetivo es la producción de madera para pulpa en rotaciones cortas, uno de los factores más importantes a considerar es la capacidad de retonación de la especie. Una especie que rebrote vigorosamente tiene claras ventajas sobre aquellas que no tienen esta capacidad, ya que permite eliminar los costos de reforestacion, y un crecimiento acelerado por varias rotaciones.

Las especies del género Eucalyptus de más interés para la forestación en Chile son E. globulus ssp. globulus; E. nitens; E. delegatensis; E. viminalis y E. regnans.

De acuerdo a los antecedentes disponibles, E. globulus ssp. globulus y E. viminalis presentan una gran capacidad de retonación, que puede dar origen a sucesivas rotaciones de monte bajo.

Las otras especies, en cambio, de acuerdo a lo que indica la literatura especializada, presentarían ciertos problemas para su regeneración desde el tocon. Esto hace que algunas compañías y forestadores prefieran $E$. globulus, aún en zonas en donde esta especie presenta problemas de adaptación, debido a las bajas temperaturas.

Turnbull y Pryor (1978) y Opie et al. (1978) señalan que E. delegatensis no rebrota bien; que en $E$. regnans esta característica es rara y que $E$. nitens puede producir regeneración de tocon, pero con ciertas dificultades.

La experiencia en Chile indica que en determinados casos las especies $E$. delegatensis y $E$. regnans rebrotan con bastante profusion y vigor; pero en otros, la regeneración es escasa o nula. Esto indica que hay factores tales como la procedencia, la 
época de corta y al parecer, la altura de corte, que estarían incidiendo en la rebrotación de estas especies.

Los árboles de $E$. nitens que han sido cortados en Chile son escasos y en su mayoría forman parte de las parcelas del proyecto de introducción de especies del Instituto Forestal. La mayoría de estos árboles han retonado, pero no muy vigorosamente. Esto puede deberse a que han quedado bajo el dosel de los otros árboles que componen la parcela.

Las parcelas de E. nitens considerados en este estudio son las primeras que se cortan a tala rasa, permitiendo observar la retonación de la especie sin la influencia de otros árboles.

El presente trabajo analiza y compara la capacidad de retoñación de estas especies, aportando información que, sin duda, es importante al momento de tomar una decisión en cuanto a la o las especies a emplear, especialmente en proyectos destinados a la producción de pulpa.

\section{MATERIAL Y METODO}

Se analiza la retoñación de las especies E. globulus ssp. globulus, E. delegatensis, E. nitens, E. regnans y E. viminalis, en parcelas de introducción de especies establecidas por el Instituto Forestal en 1968 y 1969, que fueron cortadas en Febrero de 1989 por el propietario del predio en donde INFOR había establecido el ensayo. Las parcelas están ubicadas en las cercanías de Mulchén.

Por tratarse de plantaciones experimentales, las especies están en parcelas distribuidas de acuerdo a un diseño estadístico. El diseño es de parcelas completamente aleatorizadas con tres repeticiones. En este caso, sin embargo, sólo se consideran los árboles con un desarrollo normal al momento de la corta, y que no hubiesen sido cortados anteriormente. La diferencia de edad ( 1 año) entre algunas de las parcelas se considera irrelevante para los efectos de este estudio.

La retonación se midió en Abril de 1990, es decir, 14 meses después de la corta, registrándose las siguientes variables: \% de tocones retoñados, número de retoños, altura máxima, diámetro del retoño de mayor altura, altura del tocón y diámetro del tocón. Además se registró el tipo de retoño, según su origen en el tocón. Los porcentajes se transformaron a arcosenos para su análisis estadístico. 
Con el fin de conocer si existen diferencias en la capacidad de retonación de las distintas especies en estudio, se comparan algunas de las variables registradas en terreno, mediante Análisis de Varianza. También se incluye la variable $\mathrm{D}^{2} \mathrm{H}$, que constituye una mejor estimación de la biomasa desarrollada por el retono.

Los supuestos de homocedasticidad, normalidad y aditividad se comprueban con las pruebas de Bartlett, Shapiro - Wilk y Tukey respectivamente.

Para especificar las diferencias, cuando estas existen, se emplea el test de comparación múltiple de Tukey.

Además, se pretende establecer una relación entre la capacidad de retonación y algunas características del tocón, que reflejan condiciones propias del árbol (diámetro del tocón) y del sistema de cosecha (altura del tocón), mediante análisis de regresion.

\section{RESULTADOS Y DISCUSION}

Después de 14 meses de haber sido cortados los árboles, la regeneración es abundante y vigorosa en 4 de las especies estudiadas. Solo la especie $E$. regnans presenta un porcentaje de retonación considerablemente inferior. Así lo indican los resultados que se entregan a continuación.

\section{Capacidad de retoñación}

La capacidad de retoñación está dada por el porcentaje de tocones que emitieron retoños y por el número de retoños que lograron desarrollarse. Estos resultados se presentan en el Cuadro № 1 .

\section{Cuadro № 1 \\ PORCENTAJE DE RETOÑOS Y NUMERO DE RETOÑOS, POT TOCON, SEGUN ESPECIE}

\begin{tabular}{|l|c|c|}
\hline \multicolumn{1}{|c|}{ ESPECIE } & \% DE RETOÑACION & $N^{2}$ DE RETOÑOS $(x)$ \\
\hline E. viminalis & $100,0 \mathrm{a}$ & $5,2 \mathrm{c}$ \\
E. globulus ssp. glubulus & $93,1 \mathrm{a}$ & $6,6 \mathrm{~b}$ \\
E. nitens & $81,8 \mathrm{a}$ & $5,9 \mathrm{~b} \mathrm{C}$ \\
E. delegatensis & $80,1 \mathrm{a}$ & $8,0 \mathrm{a}$ \\
E. regnans & $17,0 \mathrm{~b}$ & $4,7 \mathrm{C}$ \\
\hline
\end{tabular}


E. viminalis y $E$. globulus confirman su excelente capacidad para retoñar después de la corta. En el caso de $E$. globulus se produjeron fallas, debido a que algunos tocones quedaron tapados por desechos de la explotación, lo cual impidió una retonación normal. Esto también afectó a otras especies.

Aún cuando estadísticamente $E$. delegatensis y $E$. nitens no presentan diferencias significativas con las especies $E$. viminalis y $E$. globulus, la diferencia en cuanto a porcentaje de retonación es considerable, ya que en la práctica esto significa perder un importante porcentaje de la capacidad productiva del bosque.

$\mathrm{Al}$ analizar las causas de la falta en la retoñación en $E$. delegatensis se pudo constatar que existe una alta correlación entre este proceso y la altura del tocón. La mayoría de los árboles que no rebrotaron fueron cortados a ras de suelo. Se puede decir que todos los árboles cortados a una altura normal $(15-20 \mathrm{~cm}$.) rebrotaron sin problemas. Fuera de lo señalado, el número y desarrollo de los rebrotes no mostró correlación con las características del tocón (altura y diámetro), tanto en esta como en las otras especies en estudio.

En consecuencia, los porcentajes de retonación de $E$. delegatensis podrían ser muy superiores a los señalados en este estudio, si se tiene la precaución de dejar un tocón de unos $20-25 \mathrm{~cm}$. de altura.

En el caso de E. nitens, también debería aumentar el porcentaje de retoñación al hacer una explotación más cuidadosa.

Los resultados de E. regnans confirman que tiene escasa capacidad para retoñar, lo cual le deja en clara desventaja frente a las otras especies.

$\mathrm{Al}$ analizar el número de retoños por tocón, la especie que rebrota más abundantemente, contra todo lo señalado, es el E. delegatensis. Aún cuando estadísticamente se producen diferencias significativas entre las especies, desde el punto de vista práctico éstas no son de gran importancia, ya que todas presentan un número de retoños suficiente como para asegurar la regeneración.

\section{Tipo de retoño}

La retonación de los Eucalyptus puede producirse a partir de yemas adventicias que se forman en la parte alta del tocón, entre madera y corteza, como consecuencia de la herida producida por el corte, y de yemas latentes, epicormicas, situadas en los costados 
del tocón, que se activan debido al desbalance fisiológico que se produce al cortar el árbol (Kramer y Kozlowski, 1960).

Por lo general la retoñación de yemas adventicias es más frágil y tiende a caerse por efecto del viento, desprendiendo la corteza. Este se observa con frecuencia en la regeneración de $E$. delegatensis, que se caracteriza por una corteza bastante blanda. La retonación de yemas latentes bajas, en cambio, es más firme y es la que tiene mayores probabilidades de desarrollarse.

En el cuadro siguiente se indica el número de retoños según su origen.

\section{Cuadro № 2}

NUMERO DE RETOÑOS SEGUN SU ORIGEN

\begin{tabular}{|l|c|c|}
\hline \multicolumn{1}{|c|}{ ESPECIES } & $\begin{array}{c}\text { Ne DE RETOÑOS } \\
\text { ADVENTICIOS } \\
(\mathbf{x})\end{array}$ & $\begin{array}{c}\text { Ne DE RETOÑOS } \\
\text { EPICORMICOS } \\
(\mathrm{x})\end{array}$ \\
\hline E. delegatensis & 2,2 & 5,8 \\
E. globulus ssp. globulus & 2,4 & 4,2 \\
E. nitens & 1,2 & 4,7 \\
E. regnans & 0,1 & 4,6 \\
E. viminalis & 2,1 & 3,1 \\
\hline
\end{tabular}

En todas las especies, la mayoría de los retoños tiene su origen en yemas epicormicas, lo cual asegura la estabilidad de la regeneración.

\section{Crecimiento}

El crecimiento alcanzado por los retoños se presenta en el Cuadro №3, expresado en base a tres variables : Altura máxima, Diámetro máximo y $\mathrm{D}^{2} \mathrm{H}$, que combina las anteriores, dando una mejor idea de la biomasa total alcanzada por el retoño de mayor desarrollo. 


\section{Cuadro № 3 \\ ANTECEDENTES SOBRE EL CRECIMIENTO DE LOS RETOÑOS SEGUN ESPECIE, 14 MESES DESPUES DE LA CORTA}

\begin{tabular}{|l|c|c|c|}
\hline \multicolumn{1}{|c|}{ ESPECIES } & $\begin{array}{c}\text { ALTURA } \\
\text { MAXIMA } \\
(\mathrm{cm})\end{array}$ & $\begin{array}{c}\text { DIAMETRO } \\
\text { MAXIMO } \\
(\mathrm{cm})\end{array}$ & $\begin{array}{c}\text { D' } \\
\text { MAXIMO } \\
\left(\mathrm{cm}^{3}\right)\end{array}$ \\
\hline E. globulus ssp globulus & $330 \mathrm{a}$ & $3,2 \mathrm{a}$ & $3,806 \mathrm{a}$ \\
E. regnans & $257 \mathrm{a}$ & $3,3 \mathrm{a}$ & $3,289 \mathrm{a}$ \\
E. nitens & $236 \mathrm{a}$ & $3,4 \mathrm{a}$ & $2,707 \mathrm{a}$ \\
E. viminalis & $271 \mathrm{a}$ & $2,9 \mathrm{a}$ & $2,299 \mathrm{a}$ \\
E. delegatensis & $228 \mathrm{a}$ & $2,6 \mathrm{a}$ & $1,704 \mathrm{a}$ \\
\hline
\end{tabular}

a : Las especies acompañadas por la misma letra no presentan diferencias significativas.

$\mathrm{Al}$ analizar las variables Altura máxima, Diámetro máximo y $\mathrm{D}^{2} \mathrm{H}$, las diferencias no son estadísticamente significativas, aún cuando son de importancia. En términos de altura $E$. globulus y $E$. viminalis son las especies de mayor crecimiento, pero si se toman las dos variables combinadas, se destacan también los desarrollos de $E$. regnans y $E$. nitens, que presentan un considerable desarrollo diamétrico.

LLama la atención el menor desarrollo alcanzado por $E$. dele gatensis, aún cuando esto concuerda con el patrón que ha demostrado esta especie en Chile, que por lo general presenta un lento desarrollo en los 10 primeros años, y un desarrollo acelerado en los años siguientes.

\section{CONCLUSIONES}

E. globulus ssp. globulus y E. vinimalis presentan una excelente capacidad de retonacion y con los debidos cuidados al momento de la explotación se puede esperar que casi la totalidad de los árboles rebroten. Esto asegura varias rotaciones de monte bajo.

E. delegatensis y E. nitens, en contra de lo que señala la literatura, retoñan bien, con lo que se podría esperar por lo menos una rotación de monte bajo, antes de que el sitio quede subutilizado por una baja densidad en la plantación.

Se supone que con técnicas adecuadas de corta, se debería superar las cifras obtenidas en este estudio. 
E. regnans no presenta una buena capacidad de retonacion, lo cual le pone en amplia desventaja como especie para ser manejada en rotaciones cortas.

\section{REFERENCIAS}

Kramer, P. J. y T. Kozlowski; 1960. Physiology of Trees - McGraw - Hill Book Company Inc. $642 \mathrm{p}$.

Opie, J. E. R.A. Curtin y W. D. Incoll 1978. Stand Management. In : Eucalyptus for Wood Production. Eds. W. E. Hillis y A. G. Brown. CSIRO Australia 434 p.

Turnbull J. W. y L. D. Pryor 1978. Choice of Species and Seed Sources. In : Eucalyptus for Wood Production. Eds. W. E. Hillis y A. G. Brown, CSIRO - Australia 434 p. 